\title{
Prácticas turísticas heterodoxas y lugares sagrados. Experiencias de contactismo en la Zona Uritorco
}

\author{
Heterodox tourism practices and sacred places. Contactism experiences around \\ the Uritorco area
}

Fabián Claudio Flores

licffflores@hotmail.com

CONICET-Universidad Nacional de Luján, Argentina

Recepción: 11 Marzo 2020

Aprobación: 10 Julio 2020

Publicación: 02 Noviembre 2020

Cita sugerida: Flores, F. C. (2020). Prácticas turísticas heterodoxas y lugares sagrados. Experiencias de contactismo en la Zona Uritorco. Geograficando, 16(2), e074. https://doi.org/10.24215/2346898Xe074

\begin{abstract}
Resumen: El artículo analiza una modalidad de turismo heterodoxa y liminal vinculada al turismo ovni: las experiencias de contactismo en determinados lugares "sagrados" de la Zona Uritorco de la provincia de Córdoba (Argentina). En este sentido, las particularidades de su desarrollo, los discursos espaciales que se ponen en escena, las prácticas que se despliegan y las experiencias de los que las llevan a cabo se sitúan en una zona intersticial entre lo turístico y lo sagrado. Metodológicamente, desde un abordaje de la geografía cultural, la estrategia cualitativa con trabajo de campo, observación participante y entrevistas informales y formales a un conjunto de actores/ sujetos involucrados se complementó con el análisis visual de materiales de promoción, folletería y páginas web.
\end{abstract}

Palabras clave: Turismo, Esoterismo, Lugares sagrados, OVNI.

Abstract: The article analyzes a heterodox and liminal tourism modality linked to UFO tourism: the experiences of contactism in some "sacred" places in the Uritorco Area, province of Córdoba, Argentina. In this respect, the particularities of its development, the spatial discourses in play, the practices and experiences of who carry them out are located in an interstitial area between the touristic and the sacred. Methodologically, from a cultural geography approach, the qualitative strategy with fieldwork, participant observation and informal and formal interviews to a group of actors/subjects involved was complemented with the visual analysis of promotional materials, leaflets and webpages.

Keywords: Tourism, Esotericism, Sacred places, UFO.

"El encuentro con eventos extraordinarios que cuestionan nuestros propios cánones sobre la 'realidad' y fuerzan a cuestionarnos los supuestos ontologismos parece ser una de las expectativas más seductoras de la experiencia etnográfica, pero al mismo tiempo más resistida (y reprimida) por la antropología académica"(Escolar, 2007, p.9). 


\section{INTRODUCCIÓN}

Las formas que adoptan las modalidades turísticas parecen ser cada vez más diversas y novedosas. El crecimiento de los "turismo de nicho" y la búsqueda de experiencias turísticas "alternativas" parecen ir copando lugares y sitios que, con anterioridad, se sostenían en formas más convencionales de explotación turística.

En lo que respecta a los vínculos entre las prácticas turísticas y las dimensiones religiosas (o sagradas), ${ }^{1}$ también se ha ampliado el horizonte hacia formas menos usuales y mucho más disidentes respecto de las presentes en los esquemas convencionales del turismo religioso. Concerniente a estas morfologías peculiares (y muy diversas, por cierto), hemos denominado heterodoxias turísticas de lo sagrado a "todo un complejo universo de prácticas que se sitúa en los márgenes de lo religioso (o lo sagrado en un sentido más amplio)" (Flores y Cáceres, 2019, p. 5) y que median en la búsqueda de sujetos movilizados que construyen los lugares como sitios turísticos (y sagrados a la vez), independientemente de que estos se hayan conformado con atractivos legitimados.

La ciudad de Capilla del Monte y su entorno, en el valle de Punilla cordobés, es un verdadero laboratorio para explorar este tipo de fenómenos que se definen por su pluralidad y complejidad. Desde mediados de los años 1980, esta comarca serrana experimentó un proceso de esoterización (Otamendi, 2008) a partir de la aparición de la llamada "Huella del Pajarillo", ${ }^{2}$ que transformó los imaginarios y las prácticas turísticas de la localidad y los orientó hacia nuevas formas de organización del espacio y del turismo local, con un fuerte impulso del Estado municipal; así emergía la llamada Zona Uritorco (Otamendi, 2008).

A lo largo de las tres décadas posteriores a este suceso, las heterodoxias turísticas de lo sagrado se fueron consolidando y fueron delineando un perfil turístico singular vinculado (aunque no excluyentemente) al turismo ovni. Esto desencadenó una serie de experiencias y prácticas espaciales que pusieron en valor ciertos sitios, que los transformaron en nuevos atractivos (el cerro Uritorco, por ejemplo) y redefinieron su condición de lugares de/para el turismo.

En el presente trabajo se analiza una de las tantas formas que adquiere esta modalidad heterodoxa vinculada al turismo ovni: las experiencias de contactismo ${ }^{3}$ en distintos sitios de la denominada Zona Uritorco. En este sentido, las modalidades de su desarrollo, los discursos espaciales que se ponen en escena, las prácticas que se despliegan y las experiencias de los que las llevan a cabo se sitúan en una zona intersticial entre lo turístico y lo sagrado. En este cruce, el lugar adquiere una potencia cardinal para interpretar el caso. Allí, entonces, pondremos el foco del análisis.

Metodológicamente, el desafío implica acceder a un fenómeno geográfico muy complejo y multidimensional. La estrategia cualitativa con trabajo de campo, observación participante y entrevistas informales y formales a un conjunto de actores/sujetos involucrados se complementó con el análisis visual de materiales de promoción, folletería y webs. Si bien el enfoque desde la geografía cultural y la fenomenología articuló nodalmente la pesquisa, se sumaron aportes del "giro subjetivo" (Heelas y Woodhead, 2005) y el "giro espacial" (Lindón y Hiernaux, 2011).

$\mathrm{Al}$ respecto del trabajo de campo vale aclarar que, por un lado, se participó de una jornada de contactismo (en apartados posteriores desarrollaremos sus particularidades) con uno de los emprendedores espirituales que realiza este tipo de viaje desde hace varias décadas, que está impuesto en los imaginarios locales como una de las "experiencias válidas" (en el Cerro Alfa). Por otro lado, se entrevistó a otra decena de agencias y particulares que también realizan la experiencia en distintos sitios de la zona. A esto se sumaron entrevistas a algunos participantes individuales y familias que conformaban los tours, tanto de los que participé presencialmente como de los que no. Estas observaciones participantes y las entrevistas se llevaron a cabo en plena temporada alta estival, lo que permitió entrar en contacto con una heterogeneidad de sujetos participantes con distintos perfiles y fines. Las entrevistas tuvieron dos contextos: uno más informal en los 
viajes de ida y vuelta a los sitios, y encuentros posteriores en otros lugares del centro de Capilla del Monte o en las propias agencias de turismo.

\section{Problemas de Definición}

"Esto no es una excursión, es una experiencia", decía el canalizador Osvaldo Allie, con quien participamos de un viaje de contactismo en el Cerro $\mathrm{Alfa}^{4}$ que más adelante describiremos en detalle. Y agregó: "Yo no cobro por mi trabajo. El costo se lo deben consultar al guía que nos llevará hasta el lugar”. De su discurso, y del de muchas personas que proponen este tipo de actividades en distintos sitios de la Zona Uritorco, se desprende la primera inquietud: ¿cómo catalogar este tipo de fenómenos que se ubican en una compleja zona intersticial entre "lo turístico" y "lo sagrado"?

Es claro, en principio, que no se trata de una práctica convencional, sino que más bien se aloja en una modalidad en la cuallos sujetos buscan experiencias sagradas (o espirituales) "autenticas" y consumen lugares y actividades que se incluyen (a grandes rasgos) en el universo de la new age. Estas transformaciones en las demandas de los viajeros y la emergencia de nuevos nichos expresan una serie de mudanzas sociales y culturales propias de la contemporaneidad.

Pero además de estas mutaciones de "lo turístico", el cambio social reflejó -entre otras- una modificación en las formas del creer de sujetos y grupos. La individuación de las creencias, la desinstitucionalización de la religión, el cuentapropismo religioso (Hervieu-Léger, 1996), la hibridación y las nuevas espiritualidades (Frigerio, 2016) parecen ser algunos indicios que se imponen en las morfologías y tecnologías del creer. Por ello, este tipo de fenómenos combinan tanto los componentes sagrados como los turísticos, y en este sentido, pensar en términos de heterodoxias sagradas (Wright, 2018) de lo turístico abre un camino para interpretar geográficamente el problema y sus tensiones.

Las formas con las que se suele mencionar este tipo de prácticas son muy heterogéneas. Turismo esotérico, turismo espiritual, turismo reflexivo, turismo energético, turismo new age, turismo místico, turismo ovni, son algunas de las denominaciones específicas que los académicos utilizan para referirse a este universo de modalidades que implican una situación novedosa y marginal en el esquema de los turismos sagrados (Flores y Cáceres, 2019). Los estudios que abordan estos modos son incipientes aún; sin embargo, Alex Norman (2004), un referente del campo, menciona que

"there is, however, an emergent field of research from which it is possible to sketch provisional boundaries and make connections with other area studies for which its findings will have relevance. Further, given James' direction to search for the documents humans, we have a range of sources to draw from regarding the types of activities and experiences spiritual tourists can be found having" (Norman, 2004, p.21).

Más allá de los apelativos específicos que catalogan este tipo de actividades turísticas, hay algunos puntos a destacar. Por un lado, una regularidad que aparece en la mayoría de las definiciones se vincula a la necesidad de "vivir experiencias no convencionales" que produzcan alguna transformación o afectación personal y "auténtica", hecho que nos obliga, a priori, a situar todo este tipo de modalidades en el marco del denominado "giro subjetivo" (Heelas y Woodhead, 2005), en el que además se supone la idea de que se trata de un sujeto turístico comprometido, consciente y sensible con los entornos socioculturales y naturales donde despliega sus prácticas, y cuya búsqueda incluye una multiplicidad de opciones (económicas, sociales, espirituales, personales, etc.). Por otro lado, los destinos seleccionados se supeditan a lugares que presentan singularidades geográficas que posibilitan desarrollar ese tipo de prácticas. No debe ser cualquier lugar, sino aquellos que poseen características físicas y representaciones simbólicas que habilitan la contingencia de esas experiencias: cerros y montes con características geológicas y geomorfológicas específicas; valles y llanuras con paisajes climáticos catalogados de "energéticos" o materialidades que han sido construidas históricamente como espacios sacralizados por sociedades previas (por ejemplo, las vinculadas a las comunidades indígenas). En 
este sentido, el giro espacial nos ayuda a desentramar el complejo mundo de esos espacios de representación (Lefebvre, 1992) y leer la geografía de los lugares más allá de su apariencia material. Finalmente, buscamos resaltar la necesidad de situar estas experiencias contextualmente, poniéndolas en diálogo con otras dimensiones políticas, económicas, sociales y culturales, e identificar el variopinto conglomerado de actores que operan a lo largo de todo el proceso y la trama de relaciones en la que se insertan.

\section{CaPilla del Monte: la Construcción del lugar esotérico}

Hasta comienzos de la temporada estival del 2020, el slogan que identificaba a la ciudad era "Capilla del Monte: energía, naturaleza y misterio”. Este espacio concebido, en términos de Lefebvre (1992), cristaliza las relaciones de poder y las políticas espaciales que se imponen localmente legalizando imaginarios turísticos que proponen una cierta identidad para el lugar. La Cámara de Comercio local, en la misma sintonía, replicaba los conceptos de "naturaleza", "misterio" y "energía” para presentar el turismo capillense y su potencialidad en el contexto regional. ${ }^{5}$ Estos actores (y otros) son, de alguna manera, los encargados de hacer circular "sistemas de signos elaborados intelectualmente" para hegemonizar los espacios percibidos y vividos (Lefebvre, 1992). ${ }^{6}$ Claro que la imposición y consolidación de estos imaginarios turísticos (Hiernaux, 2002) y estas representaciones del espacio no son estáticas ni se producen de un día para otro; por el contrario, son resultado de un proceso histórico que da cuenta de relaciones de poder que le otorgan el dinamismo.

La ciudad de Capilla del Monte se ha consolidado como uno de los destinos más visitados del valle de Punilla. Ya desde el siglo XIX el lugar se puso en valor como centro turístico con la llegada del ferrocarril, que motorizó el arribo de "familias adineradas de Buenos Aires, Rosario y de Córdoba", y sobre todo de poetas y artistas que vacacionaban en las quintas de la zona (Otamendi, 2008). Sus paisajes serranos y agrestes, y su clima seco, constituían atractivos para muchas personas que sufrían enfermedades respiratorias.

A partir de 1940, y con el auge del turismo social, Capilla del Monte se transformó en un destino privilegiado de toda la región. Las clases medias y los sectores populares fueron creciendo en número y ciertos sitios de la comarca se transformaron en los principales lugares a visitar: El Zapato, Los Mogotes, La Toma, El dique Los Alazanes (construido en 1939) y Los Terrones, todos atractivos donde se ponía en valor "la naturaleza". Como complemento, la "Calle Techada", construida a mediados de los años 60, era el espacio elegido para el consumo gastronómico, las compras y los paseos nocturnos, y la Iglesia de San Antonio de Padua era el hito religioso.

A mediados de los ochenta se produjo un cambio significativo en las representaciones espaciales y el imaginario turístico de la ciudad que transformaría radicalmente el perfil económico, social y cultural de la villa serrana. Un complejo entramado de actores y relaciones operaron en la emergencia de esta nueva organización espacial: la "Zona Unitorco" (Otamendi, 2005, 2015).

El germen de estos procesos se puede rastrear en la denominada "Huella del Pajarillo" y su posterior legitimación como nueva narrativa espacial dominante. Asimismo, este hito marcó un punto de inflexión entre la manera 'tradicional y la forma actual de concebir socialmente la imagen de la localidad" (Otamendi, 2015, p. 32). La temprana participación del Estado municipal, los medios de comunicación local y nacional, y algunos emprendedores turísticos precoces catapultaron, difundieron y promovieron las nuevas representaciones espaciales.

De hecho, aunque ya existía un conjunto de experiencias, ${ }^{7}$ y "la región era considerada un centro de espiritualidad newager, a partir de 1986 este tema se institucionalizó (el intendente participó de en la difusión del hallazgo), se tematizó públicamente y se difundió, para captar un tipo de turismo específico" (Papalini, 2018 , p. 67). Esta fue una marca de distinción que operó particularizando el turismo de la localidad y diferenciándola de otros sitios del valle de Punilla. 
El impacto de estos discursos fue inmediato, y a comienzos de los 90 Capilla del Monte ya había consolidado un claro perfil vinculado al turismo ovni. Alejandro Otamendi, uno de los referentes de los estudios de esta modalidad turística, lo define como

una variante peculiar de las actividades turísticas que privilegia o se apoya en el imaginario cultural de las narrativas sobre ovnis y extraterrestres, las cuales conforman el marco de significado que guía la conducta y orienta la experiencia de los turistas en los sitios visitados. (Otamendi, 2015, p. 49).

Los lugares de destino del turismo ovni y los procesos de construcción de la atractividad están atravesados por complejas tramas sociales y culturales que necesitan ser historizadas para entender los resultados finales, y advertir cómo ese atractivo se inserta en una cartografía más amplia de zonas vinculadas a esta tipología y, a la vez, cómo dialoga con otro tipo de atractivos.

Una serie de transformaciones desarrolladas a partir del nuevo siglo XXI, y con un fuerte apoyo y presencia del sector público local en consonancia con agentes privados de diversa índole, terminaron por diagramar la esoterización de la localidad. En el plano de los atractivos, hubo un proceso de redefinición de sitios y de lugarización ${ }^{8}$ de otros nuevos. El caso más emblemático es la aparición en escena del Cerro Uritorco como atractivo privilegiado de este nuevo sistema socio-cultural. ${ }^{9}$ Así, confirmamos el proceso de asignación social (Bertoncello, 2008) e imposición de cualidades específicas que ponen en valor este elemento de la naturaleza como atractivo turístico. Junto con este, otros sitios ya existentes en el sistema turístico resignifican sus sentidos y atribuciones virando sus cualidades en sintonía con las nuevas modalidades heterodoxas, como el caso de Los Terrones, Ongamira y el Cerro Las Gemelas (e inclusive El Zapato) que ya eran lugares visitados previamente, pero que en este nuevo paradigma turístico comienzan a adquirir sentidos vinculados a sus propiedades "energéticas" y "místicas". Estos discursos conjugan la espiritualidad New Age con las creencias y mitos de los indígenas comechingones, la mitología Erks y otros relatos esotéricos de diversa índole (templarios, nazis, brujas, gnomos, entre otros).

Además, las sucesivas gestiones municipales desarrollaron acciones en la misma dirección, fortaleciendo las representaciones espaciales y las políticas públicas al respecto. Algunas medidas lo ilustran: la realización de los Congresos de Ovnilogía (desde 1999) y del Festival Alienígena (desde 2012) con apoyo y participación activa de las autoridades municipales; la imposición de la marca "Capilla del Monte, energía, naturaleza y misterio" (hoy reemplazada); la promoción del componente "ufológico" como principal matiz distintivo de la villa serrana, el impulso a una economía sustentada en esta episteme y el proceso de semiotización de la localidad (Raffestin, 1986), que comenzó a hacer visible la trasformación del paisaje local. Así, se colocó un mobiliario urbano con geosímbolos ufológicos, como extraterrestres en los bancos de la peatonal, un "platillo volador" en Plaza San Martín, el cartel de ingreso a la ciudad con un alien que da la bienvenida a los turistas, la señalización del nombre "Uritorco" en el faldeo del cerro y algunos emblemas en las dependencias municipales, como la Secretaría de Turismo Municipal. ${ }^{10}$

El sector privado, lejos de mantenerse al margen, se plegó a la ola newager y multiplicó la oferta, que estalló en cientos de emprendimientos: terapias alternativas del más amplio abanico (gemoterapia, Ayurveda, cuencos de cristal, cromoterapia, magnetoterapia, reflexología, terapias del canto, activación de chakras, bioterapia, flores de Bach), restaurantes vegetarianos, librerías esotéricas, negocios de souvenirs, viajes, lugares y experiencias (por ejemplo, hay dos museos relacionados con la temática OVNI y una pirámide energética), medios de comunicación de diversa índole (radio, prensa escrita, digitales) y otras prácticas diversas en la misma sintonía (meditaciones, yoga, tarot, cartomancia, adivinaciones, fotografía Kirlian, herbología, etc.).

Del conjunto de prácticas mencionadas (y otras tantas que seguramente se nos escapan), nos interesa describir y analizar el caso de las experiencias de contactismo llevadas a cabo en distintos lugares de la zona, que conjugan las dimensiones sagradas y turísticas y que, en gran parte, emulan y recrean (con distintos dispositivos y particularidades) los rituales de contactismo que se desarrollaron en la década de 1980 por parte del "maestro" pionero Ángel Cristo Acoglanis. 
Antes de adentrarnos en los tours de contactismos y sus singularidades, vale la pena hacer una mención al proceso que se está gestando en Capilla del Monte desde inicios de 2020 (específicamente, con el lanzamiento de la temporada estival). Si bien es precipitado hablar de un cambio en el perfil de las representaciones espaciales de la localidad, con la nueva gestión municipal que asumió en diciembre de 2019 comenzaron a delinearse algunos cambios que parecen desandar (o al menos mitigar) el proceso de esoterización. ${ }^{11}$ Dos de las medidas tomadas por el recién asumido intendente, Fabricio Díaz, resumen esta tendencia. Por un lado, el anuncio de su intención de suspender el Festival Alienígena, que provocó mucho debate con la Cámara de Comercio y otros sectores sociales capillenses, ${ }^{12}$ y con el que tuvo que dar marcha atrás por presión de un amplio sector de la comunidad. Por otro lado, y quizás más contundente, la nueva gestión decidió cambiar la imagen de la marca Capilla: quitó el lema "Capilla del Monte: energía, naturaleza y misterio" y lo reemplazó por "Capilla del Monte: pueblo Uritorco", poniendo en primer plano "la naturaleza” como principal atributo de la localidad. En consonancia, el nuevo logo (que además tiene nuevos colores) rescata dos elementos centrales: el cerro Uritorco (vaciado ahora de su contenido energético, mágico o místico) y el lagarto overo, acompañado de la frase: "Naturalmente activa". En la presentación de esta renovada imagen de la ciudad, el jefe gubernamental capillense decía: "La instalación de la marca Uritorco nos parece importante. Teníamos que asociarnos al potencial que tiene el cerro. Por eso construimos el concepto asociándolo con otras dimensiones", y la secretaria de Turismo reforzaba esta idea: "Para mí es todo un desafío afrontar este nuevo concepto turístico de Capilla del Monte, para descubrirlo todo el año. Enamorar al turista será mi primer desafío". ${ }^{13}$ En días posteriores, y en plena disputa con la comunidad, Díaz explicó en su cuenta de Facebook: "Renovamos ambos accesos a nuestra querida Calle Techada, que ahora luce colores que trasmiten alegría y diversidad, con un diseño de marca moderno y el slogan 'Pueblo Uritorco', que tiene un sentido publicitario, anclado en el cerro, que es conocido en el mundo, y la denominación 'Pueblo' que remonta al origen de nuestra ciudad". ${ }^{14}$

\section{LOS ANTECEDENTES: LAS EXPERIENCIAS EN LA DÉCADA DE 1980}

Los años previos al fenómeno del Cerro Pajarillo fueron claves para entender las formas en las que se consolidó la localidad como la "cumbre esotérica de la Argentina". Un personaje (ngel Cristo Acoglanis) y un lugar (Los Terrones) son los protagonistas centrales para adentrarnos en ese complejo entramado de donde emergieron las narrativas fundantes.

Acoglanis llegó a Capilla del Monte en 1983, con un bagaje que aglutinaba varios componentes: conocimientos de terapias alternativas, medicina china, quiropraxia, osteopatía, homeopatía y acupuntura, el agni yoga, el tantra, la meditación, los viajes astrales, lecturas relacionadas con la teosofía, el rosacrucismo, el espiritismo, el ocultismo filonazi, las teorías de la Tierra hueca y las ciudades intraterrenas, novelas de viajes al Tíbet, de profetas y transformaciones espirituales, su adhesión y militancia en el primer peronismo y una historia personal construida (y reproducida por sus seguidores) ${ }^{15}$ que legitimaba su condición de "extraordinariedad".

En su paso por otro centro energético y sagrado de Uruguay, la Estancia La Aurora ${ }^{16}$ en Salto, donde Acoglanis "aceptó" compartir su cuerpo terrenal con el de "Saruma", una entidad extraterrestre proveniente de las Pléyades, descubrió su misión:

"develar la existencia de un centro espiritual escondido en los pliegues dimensionales de otra realidad, pero localizado de todos modos bajo tierra en un lugar bien definido que se debía señalar. Su nombre sería Erks, o mejor aún Ciudad de la Llama Azul. [...] Una vez descubierta esta 'ciudad etérica', debía ser activada y mantenida abierta mediante un trabajo ceremonial" (De Filippi, 2018, p. 104). 
Luego de varias incursiones por el Uritorco y otros sitios de los alrededores, Acoglanis y dos mujeres que lo asistían identificaron "el lugar" elegido para el desarrollo de las ceremonias que le permitirían llevar adelante su misión. Se trata del paraje llamado “Los Terrones” en la Quebrada de la Luna, a 14 kilómetros de Capilla del Monte. En la actualidad, es uno de los sitios que se ofrece como atractivo turístico de la zona Uritorco y donde la singularidad del paisaje y su historia mística construyen una atractividad sustentada en esas dimensiones. En su sitio oficial se lo cataloga como un "Parque autóctono cultural y recreativo" y se adjunta el imaginario territorial: "Los Terrones, un lugar para el asombro". La web propia fortalece estos imaginarios a partir de expresar una serie de "sensaciones que experimentan las personas que visitan el lugar". ${ }^{17}$

Cuando el sanador descubrió Los Terrones y comenzó el proceso de sacralización del lugar, el predio era prácticamente desconocido para el turismo y estaba bajo propiedad de un lugareño, Ramón Verón, con quien Ángel tuvo que negociar el permiso para ascender hasta el playón septentrional al atardecer, donde llevaría a cabo sus ceremonias nocturnas. Pero, además, el acuerdo estratégico se selló con la inversión que Acoglanis hizo en el lugar: mejoró el camino de acceso para los automóviles; le regaló a Verón el tractor con el que se había abierto el sendero y colaboró con sus contactos políticos para que la energía eléctrica llegara a la zona (De Filippi, 2018). Esto favoreció la turistificación del sitio, su transformación en Parque y la puesta en valor del paisaje mediante el cobro de una entrada (no para quienes participaban del ritual nocturno).

En el holograma espacial ${ }^{18}$ (Lindón, 2007) de la cotidianeidad capillense de Acoglanis aparece otra estación fundamental: el Hotel Roma, en el centro de la ciudad. Este emprendimiento era (y sigue siendo) propiedad de la familia Allie, con la qu eÁngel se vinculó rápidamente. Acoglanis estableció allí su "centro de operaciones" en los albores de la democracia alfonsinista. Hoy es un lugar que refiere a esos orígenes esotéricos de la localidad y está teñido de muchas de las representaciones espaciales que configuraron esa temprana burbuja New Age que tiñó la comarca. De hecho, sus actuales propietarios alimentan la lugarización del predio con toda la mística que legitimó el paso de "Saruma" (y otros referentes) por sus habitaciones. En su sitio web se narra cómo su fundador, Tulio Allie, tomó la decisión de llevar adelante el emprendimiento: "Miré el cerro y sentí su conexión, era como si me hablara una energía única; de esta manera Capilla se convirtió en su lugar en el mundo". ${ }^{19}$ Fortaleciendo estos imaginarios, en el salón comedor una frase sentencia: "1917-2017: un siglo hospedando a los referentes del misterio".

Las ceremonias de contacto en Los Terrones se iniciaron en marzo de 1983 y se llevaron a cabo hasta 1989, cuando Acoglanis fue asesinado en su consultorio de Buenos Aires. En un principio, el ritual estaba encapsulado entre unos pocos seguidores que conformaban la trama social en la que se encontraba inmerso Acoglanis, pero de a poco esto se fue ampliando a partir del "boca a boca", que operaba como transmisor de la experiencia que se desarrollaba cada fin de semana.

Paul Ricoeur señala que "se precisan relatos y ritos para consagrar el contorno de los signos de lo sagrado: lugares santos y objetos sagrados [...]” (Ricoeur 2004, p. 318). Es decir, tanto la práctica como la representación espacial son -de alguna manera- los activadores de esa textura de lo sagrado que adquieren determinados lugares. Entonces, la selección de Los Terrones en tanto lugar sagrado (y veremos que luego de la muerte de Acoglanis hay otros nuevos) funciona en el marco cultural y espacial de las ceremonias de contactismo y la intersubjetividad de sus participantes.

La primera parte de la experiencia comenzaba al atardecer en el playón de Los Terrones, cuando Ángel Cristo Acoglanis daba una charla con los principales contenidos de la cosmología Erks a los pocos asistentes que se sentaban en círculo. Luego, en la apertura, se colocaba una especie de túnica blanca y comenzaba a recitar una serie de mantras en irdin (el lenguaje extraterrestre) acompañado de dos sacerdotisas: Graciela Beatriz Münh (su esposa) y Marisa Mur (su asistente). Ese momento constituía un rito de pasaje por el que Acoglanis pasaba a ser Saruma, "quien extendía los brazos al cielo e invocaba a los Hermanos del Cosmos” (Dangel, 2012, p. 14) pidiendo autorización para comenzar. 
A partir de allí se ponía en escena el contactismo en sí mismo, en el cual Saruma se comunicaba con las luces de diferentes colores, intensidades y formas que se veían en distintos puntos del valle y a las que identificaba como las energías de las entidades superiores. Además, el canto en irdin del canalizador establecía un "diálogo" que hacía que "las luces estén atentas a cuanto hiciera, dijera y cantara Saruma, que le otorgaba un nombre a cada uno" (De Filippi, 2018, p. 110).

La segunda parte del ceremonial implicaba moverse en automóvil hasta un nuevo playón, en una zona más abierta desde donde se observaba el valle, el Cerro Uritorco y el Pajarillo. Se retomaba el ritual con los mantras y se continuaba visualizando las energías, pero a una distancia menor. Allí, y solo esporádicamente, Saruma activaba la posibilidad de visualizar la ciudad de Erks. "La emoción general era tal que en ese punto alguien podía desvanecerse y era socorrido enseguida por Acoglanis" (De Filippi, 2018, p. 110).

La tercera y última parte, luego de desplazarse un poco más hasta donde había un algarrobo aislado, se iniciaba cuando el maestro avanzaba por el camino oscuro y en soledad para "encontrarse con los Hermanos Superiores y hablar con ellos personalmente", ${ }^{20}$ y desaparecía por un lapso de 20 a 30 minutos. Los participantes aguardaban en estado de meditación hasta que regresaba Saruma con un mensaje de la Jerarquía Espiritual para los asistentes en esa jornada.

A pesar del número reducido de participantes, algunos testimonios dispersos en las exiguas fuentes y relatos de la época ${ }^{21}$ dan cuenta de la ocasionalidad con la que ocurrían situaciones atípicas, como el descenso de naves, la presencia de aroma a geranios, el sonar de coros de fondo, la aparición de animales extraños (y hasta seres extraterrestres) y la emergencia de luces azuladas provenientes del interior del Templo de la Esfera de Erks.

El ritual concluía con cantos repetitivos en castellano que entonaba Saruma agradeciendo a los Hermanos Superiores, y delos que participaba el resto de los concurrentes en el "altar mayor" de Los Terrones. Al quitarse la vestimenta, Saruma volvía a su "ser terrenal" que mechaba sus jornadas entre Córdoba y Buenos Aires, donde seguía atendiendo pacientes en su consultorio de terapias alternativas. El ritual debía repetirse durante tres jornadas con los mismos asistentes.

Estas experiencias de contactismo, regenteadas por Acoglanis, se llevaron a cabo en el mismo sitio por más de un lustro y participaron de ellas cientos de personas, que a través de sus lazos directos con el sanador o de terceros vinculados con este fueron testigos de la ceremonia protocolar que mantenía, siempre, la misma estructura. No es objeto de este trabajo conocer el entramado de mujeres y varones que participaron de estas experiencias, pero sí considerar que, en los primeros años, el grupo era muy reducido y casi secreto, y los contactos directos eran clave para ingresar. En dos años, esta red se amplió y se fueron sumando nuevos participantes. La composición era ampliamente heterogénea, pero podemos identificar un núcleo duro conformado por todos aquellos que estaban -de alguna manera- insertos en ciertas redes New Age que compartían sistemas culturales, como el caso de Beba Martel (musicoterapeuta y canalizadora); Ángeles Azcurra (especialista en terapias alternativas); la familia Allie (aficionada a las prácticas esotéricas); Yaco Albala (maestro espiritual) y su sobrino Marcelo Abdala (canalizador); Indra Devi (maestra yogui), Pedro Romaniuk (parapsicólogo); Fabio Zerpa (ufólogo) y Guillermo Terrera (docente y esotérico). Otro grupo estaba conformado por la red que nucleaba profesionales de la salud y pacientes ligados al consultorio de Buenos Aires (como el Dr. Carlos Fiore o el médico Florencio Escardó, amigo personal del sanador).

Tras el asesinato de Ángel, la situación -como era de esperarse- se complejizó. La disputa por el linaje y la continuidad del ritual en Los Terrones fue uno de los puntos centrales del nuevo panorama. La posta quedó en manos de su última mujer y sacerdotisa asistente, BetyMühn, junto a Oscar, el hijo de Acoglanis (quien tomó el lugar de Saruma) y una tercera asistente, que en primer término fue la mujer de Oscar y luego de su separación fue reemplazada por Lina Castro, una esotérica de Los Cocos que comenzó a tener protagonismo en el rubro.

Pero más allá del cambio en los actores que continuaron con la experiencia en este altar mayor es interesante destacar que tras la desaparición del gran maestro comenzó un sigiloso y acelerado proceso de puesta en 
valor del lugar y de la ceremonia, que dio inicio a un "proto turismo esotérico". En primer lugar, Bety Mühn decidió abrir, en el predio que poseía cerca de Los Terrones, una casa de té y habitaciones para alojar a los primeros turistas que deseaban quedarse cerca del lugar sagrado para realizar la práctica ceremonial que la propia anfitriona y sus ayudantes continuaban llevando a cabo. En segundo lugar, en 1992 Verón (dueño de Los Terrones) transfirió la propiedad a sus hijos Aldo y Ariel, quienes comenzaron a cobrar el ingreso a los visitantes nocturnos. Este proceso de privatización se aceleró con la muerte de Verón padre, en 1999, y con el aumento de los valores y las condiciones que impusieron sus hijos por el usufructo del espacio con fines esotéricos. Esto llevó a que el dúo Mühn-Acoglanis hijo decidiera poner fin a los 17 años ininterrumpidos durante los cuales la experiencia de contactismo se había desarrollado en el altar mayor de Los Terrones, y hacia el año 2000 dejaron de ir a ese sitio.

En tercer lugar, a mediados de los noventa la disputa por la "herencia espiritual" de Acoglanis estalló en varias propuestas, que se tradujeron en la emergencia de nuevos oferentes y en nuevos sitios sagrados que brindan las experiencias con algunas variantes alternativas.

La construcción de estos lugares sagrados, "alternativos" al altar mayor de Los Terrones, surgió a partir de contactos telepáticos de los Hermanos Superiores con algunos de los nuevos canalizadores que se desglosaban de la experiencia acoglaniesca. Así, para fines de los 90, la cartografía esotérica de la Zona Uritorco identificaba estas hierofanías emergentes:

a) Puertas del Cielo: un sitio de libre acceso localizado a un kilómetro de Ongamira y 14 de Los Terrones. Es uno de los más alejados de toda la zona y desde él se posee una vista completa del valle, el Uritorco y los cerros vecinos. Allí, el hijo de Acoglanis comenzó las experiencias luego de separarse del emprendimiento que había abordado con Bety Mühn.

b) Las Gemelas: cerro vecino del Uritorco a $6 \mathrm{~km}$ de Capilla del Monte, que en términos de representaciones concentra la energía femenina. Desde los años 90 le fue comunicado a Lina Castro telepáticamente como lugar sagrado para los rituales. Castro comenzó a ofrecer paquetes con alojamiento en Los Cocos (es hotelera de allí), con régimen de pensión de comidas vegetarianas, visitas guiadas para meditar y avistar ovnis. Luego se fue a Cuchi Corral.

c) Cuchi Corral: situado a $25 \mathrm{~km}$ de la localidad de Capilla, cerca de La Cumbre. Los imaginarios sobre el lugar refieren a la espiritualidad comechingona y la presencia de energía de Erks. Se catapultó como altar alternativo para el contactismo luego del fin de Los Terrones.

d) La tercera estación: es el primer sitio alternativo que emergió en el predio "IkiShamuaika- El lugar elegido", donde vivían Bety y Acoglanis en el cruce de las rutas 38 y 17 que conduce a Los Terrones. También fue indicado telepáticamente a la propia "Guatuma" (nombre de la entidad superior de Bety), quien realizó allí las experiencias de contactismo de forma gratuita (o con pago voluntario) hasta fines de los 2000 .

A partir del siglo XXI comenzaron a multiplicarse las experiencias de este tipo y aparecieron otras renovadas; también se incrementaron los lugares donde llevarlas a cabo, se profesionalizó la actividad y surgió un entramado de actores que, en disputa material y simbólica, ofrecen variantes diversas y nuevos destinos, como el Cerro Alfa y el de la Cruz (en San Marcos Sierras), las Cuevas de Ongamira y el Cerro Colchiquí, $\mathrm{y}$ varios predios privados en las afueras de Capilla.

\section{LAS EXPERIENCIAS EN LA ACTUALIDAD}

En las últimas décadas, la oferta turística de Capilla del Monte se ha ampliado notablemente, apoyada en la consolidación del imaginario turístico de "energía, naturaleza y misterio", y las experiencias de contactismo (en todas sus variantes) son unas de las más elegidas por los visitantes. Son ofrecidas por distintos actores que podemos nuclear en dos grupos: 
a) Agencias de turismo: la mayoría de ellas se encuentran en la Calle Techada (la principal) y ofrecen una variante más relacionada con el tour convencional. Una de las agencias más importantes de la localidad, Turismo Tres Cerritos, brinda un abanico de modalidades que incluye el rubro "Turismo Místico". Se promocionan seis propuestas: "Posta del silencio" (\$979), "Vigilantes del cielo" (\$650), "Sillón de sanación" (\$759), "Los Terrones" (\$1450), "Puertas del Cielo" (\$979) y "Leyendas y misterios del Edén" (\$715). La agencia Punto Uritorco ofrece mayoritariamente paquetes turísticos al exterior y a algunos destinos del país. A nivel local realiza excursiones más relacionadas con el "turismo aventura", excepto "Experiencia en Puertas del Cielo" con meditación con cuencos de cristal (\$900), y también un combinado que suma la visita a la Grutas de Lourdes y Ongamira para luego ver el atardecer en Puertas del Cielo con meditación y sonidos de cuencos. Y mencionan: "en nuestro city tour por Capilla del Monte, recorremos los puntos más importantes y contamos toda la historia, mitos y leyenda el lugar”, los precios van desde $\$ 400$ el citytour a $\$ 1100$ Ongamira + atardecer en puertas" ${ }^{22}$ Excursiones Angeluz es una empresa familiar que se dedica a fomentar el turismo regional y dentro de una amplitud de ofertas propone "paseos místicos". Uno de los que brindan es a Puertas del Cielo con un canalizador. Se hace de noche (a las 21) e implica un "trabajo de vaciamiento interior que lleve a soltar para dar lugar a lo nuevo". Se proponen como actividades la observación del cielo y armonización con cuencos de cristal "en un portal energético conocido como la ciudad de los Erks"; otra de las posibilidades es la visita al campo ancestral "La Victoria" combinando "naturaleza, meditación y atardecer" en "un lugar único de Córdoba" a 11 kilómetros de Capilla del Monte, "rodeado de los míticos cerros que habitaban los comechingones ideal para la conexión con la naturaleza y su mágica energía para encontrarnos con nuestro Maestro interno". ${ }^{23} \mathrm{En}$ otra dirección, y alejándose de la vertiente mística, Latinoimaginarios ofrece viajes especializados en Astroturismo. La propuesta está vinculada con la Secretaría de Turismo Municipal y realizan salidas nocturnas todos los jueves a las 21 horas, previa charla sobre conocimientos generales de la astronomía cultural (cuesta \$ 500). Se menciona como una experiencia “única” en toda la provincia de Córdoba, que se desarrolla en un espacio de las afueras de la ciudad llamado "Villa Cielo". ${ }^{24}$

b) Emprendedores espirituales: el colectivo que se encuadra en esta tipología es diverso y muy complejo. La forma de acceder a las propuestas no tiene la visibilidad que poseen las agencias de turismo que, como mencionamos, se sitúan en la zona más transitada por los visitantes. Para llegar a conocer y participar de estas experiencias funcionan el "boca a boca", las redes de lugares que se insertan en este tipo de circuitos esotéricos (por ejemplo, folletería que se halla en negocios de souvenirs, librerías esotéricas, comercios newager, etc.) y la publicidad a través de redes sociales, blogs y webs. Algunos de los casos que pudimos rastrear en el trabajo de campo dan cuenta de la heterogeneidad del grupo:

- Daniel Brower: es cuenquero y hace caminatas al Templo Zen. Es fundador del "Círculo de Sonido" y hace las "Ceremonias Mundiales de Armonización” en los solsticios y equinoccios. Actualmente realiza retiros espirituales "En equilibrio y Armonía” para los que ofrece paquetes místico-esotéricos que incluyen alojamiento con pensión completa vegetariana, con todas las actividades, incluidas las ceremonias, por $\$ 14.250$ por 4 días.

- Lina Castro: canalizadora y hotelera de Los Cocos que realiza este tipo de experiencias en Cuchi Corral. Hace 45 años que lleva a cabo este tipo de actividades y su historia, como mencionamos, está relacionada con el linaje de los Acoglanis. Hace viajes desde Los Cocos y conecta en el Cuchi Corral con esferas de cristal. Para participar no se debe comer carne, consumir alcohol ni cigarrillos el día en que se participa de la experiencia; el costo de es $\$ 1500$ pesos.

- Ariel Pro realiza excursiones místicas a Puertas del cielo, Los Terrones y Vigilancia del Cielo. Los precios van de $\$ 600$ a $\$ 1500$. Tiene sede en un local llamado "Erks" en la Galería 
Giovanni. La experiencia "Vigilancia de los Cielos" la desarrolla en el fondo de su casa, cerca del Uritorco.

- Marcelo Abdala (discípulo de Acoglanis) es el único que hace la experiencia en Los Terrones; en la actualidad, los días miércoles y sábados. El viaje parte de Las Gemelas y cuesta $\$ 1500$.

- Osvaldo Allie es uno de los propietarios del emblemático Hotel Roma, que tiene toda una tradición esotérica, y participó de las ceremonias en Los Terrones con el maestro Saruma. Se ofrece la experiencia de canalización en el Cerro Alfa y el costo es de \$ 800 .

- Ezequiel Martínez realiza caminatas de "conexión con la naturaleza”, Sabiduría Ancestral y Mindfulness, con precios que varían según la cantidad de grupos y los días.

Sumado a la presencia de diversos actores que organizan las experiencias, el panorama general nos muestra la consolidación de lugares sagrados (y alternativos al altar mayor de Los Terrones) y la emergencia de algunos nuevos. En todos los casos, estos sitios funcionan como escenarios ideales para que la experiencia sea efectiva y están atravesados por discursos que combinan sus propiedades naturales (la composición de minerales y rocas, la amplitud para visibilizar el paisaje, la altura de los cerros) con las condiciones extraordinarias que les fueron adjudicadas mediante procesos de imposición culturalmente definidos (la presencia de energías extraterrestres, la existencia de antepasados originarios y rituales sagrados o sucesos específicos que activaron esa sacralidad). Por ejemplo, el Cerro Colchiqui, uno de los actuales lugares sagrados para el contactismo, funda sus propiedades en una leyenda que refiere el suicidio en masa de mujeres, niños y ancianos que se arrojaron para evitar ser sometidos por los españoles; como nunca se encontraron sus cuerpos, se alude a que pasaron a otra dimensión. Esta narrativa opera como garante de las propiedades del lugar para desarrollar la práctica eficazmente.

A grandes rasgos, y con algunas diferencias, las experiencias de contactismo en la actualidad mantienen una estructura bastante similar al modelo que Acoglanis desplegó en la década del 80. De hecho, la similitud de su morfología y el linaje del canalizador o canalizadora operan como garantes de su autenticidad, siempre considerando que ésta es resultado de un proceso social y cultural de puesta en escena (McCannell, 2003). Tarlow \& Mills (1998), que han analizado el caso del turismo extraterrestre en torno al Área 51 y Roswell (ambos en Estados Unidos), expresan que, en estos casos, "una no-realidad es mostrada como si fuese real convirtiéndola en una realidad turística efectiva” (Tarlow \& Mills, 1998, p. 266), que se traduce en hotelería, souvernirs, gastronomía, agencias, tours, guías, etc. Pero en la construcción de la autenticidad (al igual que en la de la atractividad) operan -además- dimensiones políticas, económicas, sociales y culturales, independientemente de su configuración y morfología.

"Al fin y al cabo la autenticidad buscada por el turista no necesariamente tiene que coincidir con la materialidad forjada en un área. La autenticidad tiene más que ver con el cómo se percibe una experiencia y/o artefacto (qué valores admirables se contemplan encarnados en ellos y con qué estética son expresados) que con la cosificación de la experiencia y el artefacto mismo" (Santana Talavera, 2002, p. 15).

Y en este sentido, todos los participantes con los que asistimos a la experiencia en el cerro Alfa (10 personas) expresaron de diferentes formas "su satisfacción" por haber podido experimentar esa "autenticidad" gracias a dos componentes: el lugar y el canalizador. Del primero se resaltaba "la energía del lugar”, "el misticismo y la paz", "la vista imponente que te transporta", "una especie de energía que no te deja salir del cerro"; ${ }^{25}$ del segundo, la experticia, experiencia y linaje del contactista son clave. El tour lo realizamos con un reconocido guía local y aficionado al esoterismo (Larry Roldán, propietario de la Pirámide del Uritorco) y la ceremonia fue dirigida por Osvaldo Allie, dueño del Hotel Roma y copartícipe de las ceremonias en Los Terrones desde los primeros años. Cabe aclarar que, antes de la partida hacia el lugar sagrado, tuvimos una charla previa de una hora en el salón principal del Hotel Roma, donde Allie narró su historia personal con Acoglanis, su experiencia en Los Terrones y gran parte de las explicaciones "teóricas" sobre Erks, contactismo, energías y otras ideas que íbamos a "necesitar" saber durante la experiencia. Retomando la idea de autenticidad de la 
experiencia, cabe aclarar que, más allá de las distintas ofertas y agentes, la escenificación es fundamental para sudesarrollo: desde el clima que se genera, el tono de voz y los movimientos del canalizador hasta las acciones propuestas y la dimensión performativa. Esta escenificación implica desplazamiento de producción cultural de un lugar para otro, pero no necesariamente superficialidad (Chhabra, 2005). 'El 'producto' consumido finalmente puede no ser 'tradicional' para el grupo visitado, pero lo construido artificialmente aparece ante la mirada del turista/visitante como más real que lo real mismo" (Saarinen, 1998), porque después de todo la autenticidad también es creada por el turista/visitante (Delyser, 1999).

Retomando la estructura de los tours, en la mayoría de los casos se comienza con algún tipo de charla para poner en tema. Este discurso inicial funciona no solamente para conocer los marcos culturales en torno a los que se desarrollará la experiencia, sino también como regulador de las prácticas permitidas y vedadas en el marco del ritual y de las propiedades de sacralidad de los lugares que serán escenarios. Ya instalados en el sitio, la experiencia comenzó cuando el canalizador realizó los mantras de apertura (en irdin) para solicitar "permiso" a las Jerarquías Superiores e iniciar el contactismo. En la mayoría de los casos los grupos suelen ser reducidos (no más de 12 personas) y hay uno o dos líderes que "facilitan" la experiencia. Esta dura unos 50 a 60 minutos e incluye el avistaje de naves y de las entidades superiores (todo bajo la forma de luces), y, en ocasiones excepcionales, alguna referencia de la visualización de Erks. El canalizador realizó la práctica de contacto "dialogando" con las luces e intercambiando mensajes con una linterna láser, y traduciendo a los asistentes quiénes eran las entidades que se iban haciendo presentes (con los nombres correspondientes a la cosmología de Erks: por ejemplo, Saruma, Guatuma, Sikuma,etc.). Luego de la observación y decodificación de la coreografía lumínica y de los sitios por donde se encuentran y desplazan los Seres Superiores, el contactista recibió "un mensaje" en irdin para los asistentes y lo fue traduciendo simultáneamente. Algunas personas manifestaron un estado de "asombro", "satisfacción plena”, “conmoción”" ${ }^{26}$ entre otras emociones en esa dirección. Esa emocionalidad se expresaba de distintas formas: desde llanto, contemplación, silencio hasta una persona que se desmayó y fue asistida por el canalizador. Después hubo unos 15 minutos de tiempo libre, durante los cuales algunos meditaron, otros rezaron, otros permanecieron en silencio observando el cielo. Mientras tanto, el canalizador se acercó a hablar individualmente con algunos "elegidos" y les expuso el mensaje personal que los seres de luz le enviaban a cada uno de ellos. Hubo, sobre el final, tiempo para fotos y videos y el cierre fue con los mantras finales del guía espiritual, agradeciendo la experiencia.

Dos consideraciones son pertinentes a la hora de pensar la liminalidad en la que se sitúa la experiencia. Por un lado, la notable diferencia de formas y sentidos que se establecen entre las que son organizadas por las agencias y las que se llevan a cabo por los emprendedores espirituales: el marco del ritual, las formas de organización del viaje y las prácticas hacen que en los primeros prime una cierta lógica más "turística" y que el tour se inserte en una más de las posibilidades de viajes ofrecidos en el destino. En las segundas, se pone en primer lugar la dimensión ritual y sagrada por encima de la turística, aunque esto no quita que "en todos estos casos, el viaje, si bien se envuelve dentro de un marco de significados muy particular, al mismo tiempo, adopta las formas de los tours tradicionales, indicando la secuencia de actividades para los distintos días y momentos, propios de la modalidad turística" (Otamendi, 2008, p. 189).

Por otro lado, dentro del grupo de los emprendedores hay una enérgica referencia tanto a Acoglanis como a sus ceremonias en Los Terrones y la disputa por la herencia de "su legado". La mayoría de ellos replica y resignifica los pasos de la ceremonia, dándoles su toque personal (por ejemplo, música de cuencos, una ceremonia de esferas de cristal, meditación, etc.) y poniendo en escena una performatividad y una emocionalidad que evoca esos tiempos/espacios. Esto no está exento de disputas, no solamente por la autenticidad y efectividad de la experiencia, sino también por las "anomalías" que fueron surgiendo a partir del crecimiento del número de emprendedores que, sin haber sido testigos y protagonistas de aquellos días, se suman a ofrecer este servicio.

Finalmente, y respecto de la dimensión espacial, es notable la ampliación de sitios donde se practica la experiencia contactista (como hemos descripto), pero además, si bien los procesos de sacralización de estos 
lugares se apoyan en las representaciones espaciales previas (relatos místicos y esotéricos), también influyen los sentidos que adquieren en el curso mismo de la ejecución de las prácticas, que suelen transferir a los lugares ese tipo de significados. Siguiendo a Lindón (2017):

"las prácticas siempre proceden de tramas de sentido, reactivan sentido y reconstruyen significados, pero todo ello está mediado por su dimensión espacial: los lugares en los que ocurre la vida práctica les dan sentido a las prácticas, y las prácticas reconstruyen el sentido de los lugares." (Lindón, 2017, p. 112).

\section{Conclusiones}

El análisis de los fenómenos que combinan lo turístico y lo sagrado parece encontrar un horizonte muy fructífero más allá del clásico esquema del turismo religioso y de sus dicotomías funcionales. La idea de experiencias heterodoxas y liminares nos permite ampliar la mirada y poner en tensión conceptos y formas para captar la complejidad de fenómenos que parecen tener cada vez más presencia en el universo del turismo.

Capilla del Monte y su historia espacial de las últimas cuatro décadas está fuertemente ligada a los discursos esotéricos y las narrativas ovni que permearon enérgicamente en gran parte de los antiguos y nuevos pobladores de la comarca. Esta "marca de distinción" operó como articuladora de todo un sistema cultural que generó una dimensión turística y económica en crecimiento.

Estas heterodoxias turísticas de lo sagrado adquieren diversas formas y denominaciones que apuntan a poner el foco en el perfil de los que las practican y la subjetividad que ponen en juego en esas instancias. Norrild (1998) prefiere hablar de turismo místico-esotérico para referirse al conjunto de actividades turísticas que conjugan la búsqueda espiritual de quienes se desplazan, y adiciona a la presencia del componente sagrado un elemento que le da sustancia: el rito de iniciación (Norrild, 1998). En esta tipología, el perfil de los visitantes está caracterizado, según la autora, por sujetos que, en general, "cuentan con alguna preparación previa conocida como rito de iniciación" (Norrild, 1998, p. 236) y están insertos en tramas socio-culturales más amplias. Sin embargo, algunas investigaciones más actuales (Haq \& Jackson, 2006; Norman, 2012; Flores y Cáceres, 2019) dan cuenta de la existencia de turistas que no sólo no cuentan con ese conocimiento previo, sino que también combinan el consumo de esos lugares con otros fines, y la espiritualidad es uno más. Se trata de algo similar a los "casual spiritual tourists", "incidental spiritual tourists" y" serendipitous spiritual tourists” de la clasificación de Haq y Jackson (2006). Para el caso de las experiencias de contactismo el panorama es muy amplio y, si bien hay algunos visitantes que llegan con algún background New Age o insertos en redes más sólidas, hay un grueso de curiosos que lo hacen motivados por la necesidad de consumir el marketing de la experiencia extraordinaria; para ellos, esa visita, se transforma en un destino más dentro de un abanico de lugares y actividades por desarrollar. Asimismo, a nivel local, desde el sector turístico se utilizan términos como alternativo, viajes de conexión o de contacto, ovni, encuentros programados, jornadas especiales de armonización y contacto, turismo espiritual, esotérico y mistico, entre otras denominaciones (Otamendi, 2015).

Tanto en unos como en otros opera un conjunto de condicionantes y circunstancias que hacen que la experiencia se vuelva un hecho "auténtico". En esta situación,

“el turista proyecta en los objetos y el lugar estereotipos, fantasías e ideologías. La experiencia se torna auténtica si se consigue establecer una conexión entre lo que el turista proyectó y lo que vivencia, independientemente de si los objetos y los lugares son legítimos" (Pérez Winter, 2013, p. 788).

Y allí el éxito se torna una matriz frecuente en la mayoría de las experiencias.

Finalmente, vale la pena resaltar el rol del abordaje geográfico-cultural para comprender la complejidad de procesos y lugares que dan origen a prácticas turísticas y sagradas que no responden a las formas más convencionales en las que se expresa el turismo contemporáneo, en la medida en que los marcos culturales son claves para entender cómo funcionan estos lugares y procesos, más allá de las dimensiones económicas o políticas. Además, se hace necesario poner en contexto histórico, político y social la presencia de procesos 
tan dinámicos e inestables como las direcciones, prácticas y representaciones en que se sustentan las políticas turísticas. Prueba de ello es que, al momento de culminar este artículo, la política turística local (con grandes tensiones y rechazos) intentaba virar el perfil esotérico de la localidad cordobesa.

\section{REFERENCIAS}

Agnew, J. (1993). Representing space. Space, scale and culture in social science. En J. Duncany Ley, D. (Eds.), Place/ culture/representation (pp.1-26). Londres: Routledge.

Bertoncello, R. (2008). Presentación. En R. Bertoncello (Comp.),Turismo y Geografía. Lugares y Patrimonio naturalcultural de la Argentina (pp. 5-15).Buenos Aires: Ciccus.

Chhabra, D. (2005). Defining authenticity and its determinants: Toward an Authenticity Flow Model. Journal of Travel Research, 44, 64-73. https://doi.org/10.1177/0047287505276592

Dangel, G. (2012). Todo sobre el cerro Uritorco y la ciudad de Erks. Bs. As.: de la Tortuga.

De Filippi, S. (2018). La ciudad de la llama azul. Luces y sombras sobre el Cerro Uritorco. Buenos Aires:Biblos.

Delyser, D. (1999). Authenticity on the Ground: Engaging the Past in a California Ghost Town. Annals of the Association of American Geographers, 89(4), 602-632. https://doi.org/10.1111/0004-5608.00164

Escolar, D. (2007). Lights in Calingasta Valley. Reality and theethnography of the extraordinary. International Earthlights Alliance (IEA). Recuperado de: http://www.earthlights.org/scholarlycontributions.htm

Flores, F. (2011). ¿Turistas o peregrinos? Prácticas en torno al fenómeno religioso en San Nicolás de los Arroyos. Transporte y Territorio, 5, 72-88. https://doi.org/10.34096/rtt.i5.267

Flores, F. (2016). Espacialidad y religiosidad: encuentros y desencuentros teórico-metodológicos. Cultura y Religión, 10(1), 3-16. Recuperado a partir de https://www.revistaculturayreligion.cl/index.php/culturayreligion/article /view/630

Flores, F. (2018). Lo religioso y el espacio. Apuntes desde la Geografía. En A. R. Cloclet da Silva y R. Di Stefano. (Coords.), História das Religióes: dimensões epistemológicas e teórico-metodológicas (pp. 201-242). Rio de Janeiro: Prismas.

Flores, F. y Cáceres, C. (2019). "Heterodoxias turísticas. Lo exótico y misterioso como atractivo turístico en los valles Calchaquíes de Salta”. Posición, 1, 1-24. Recuperado a partir de https://716132a6-9cf5-45de-baee-6a15e46210 f7.filesusr.com/ugd/df634b_6c79500d474a44edb17c2bdbe29c6a25.pdf

Frigerio, A. (2016). La ¿"nueva”? espiritualidad: ontología, epistemología y sociología de un concepto controvertido. Ciencias Sociales y Religión, 18(24), 209-231. Recuperado de https://econtents.bc.unicamp.br/inpec/index.php /csr/article/view/12604/7979

Haq, F. \& Jackson, J. (2006). Exploring consumer segments and typologies of relevance to spiritual tourism. Paper presented at Australia and New Zealand Academy of Marketing Conference. Brisbane, Australia, Queensland University of Technology.

Heelas, P. \& Woodhead, L. (2005). The spiritual revolution: Why religion is giving way to spirituality. Oxford: Blackwell Publishing.

Hervieu-Léger, D. (1996). Catolicismo, el desafío de la memoria. Sociedad y Religión,14, 1-25.

Lefebvre, H. (1992). The production of space. Cambridge: Blackwell.

Lindón, A. (2007). Los imaginarios urbanos y el constructivismo geográfico: los hologramas espaciales. EURE, 23(99), 31-46. https://www.doi.org/10.4067/S0250-71612007000200004

Lindón, A. (2017). La ciudad movimiento: cotidianidades, afectividades corporizadas y redes topológicas. Inmediaciones de la Comunicación, 12(1),107-126. https://www.doi.org/10.18861/ic.2017.12.1.2668

Lindón, A. yHiernaux, D. (Dirs.) (2011). Los giros de la geografía humana. Desafíosy horizontes. Itzapalapa: AntrophosUAM. 
Massey, D. (2004). Lugar, identidad y geografías de la responsabilidad en un mundo en proceso de globalización. Treballs de la SocietatCatalana de Geografia, 57, 77-84. Recuperado de https://publicacions.iec.cat/repository/ pdf/00000019/00000025.pdf

Norrild, J. (1998). Turismo y Esoterismo: una aproximación al tema. Estudios y Perspectivas en Turismo, 7(3-4), 235-249.

Norman, A. (2012). The varieties of the spiritual tourist experience. Literature \& Aesthetics, 22(1), 20-34. Recuperado de https://openjournals.library.sydney.edu.au/index.php/LA/article/view/7573

Otamendi, A. (2008). El turismo místico-esotérico en la Zona Uritorco (Córdoba): Síntesis de una perspectiva etnográfica. Revista Brasileira de Pesquisa em Turismo, 2(2), 20-40. Recuperado de https://www.redalyc.org/p df/5041/504152238003.pdf

Otamendi, A. (2015). El viaje interno. Simbolos, narrativas y turismo ovni en creyentes de los extraterrestres de la Argentina. (Tesis doctoral). Buenos Aires, Facultad de Filosofía y Letras de la Universidad de Buenos Aires. Recuperado de http://repositorio.filo.uba.ar/handle/filodigital/5987

Papalini, V. (2018). Sincretismo de la new age sudamericana: una amalgama sin integración. El caso de Capilla del Monte, Argentina. Revista ScriptaEthnologica, 40, 63-84. Recuperado de https://ri.conicet.gov.ar/bitstream/handle/11336/92540/CONICET_Digital_Nro.e765022a-f78b-432b80b1-31c9aa8408cc_A.pdf?sequence=28isAllowed $=y$

Pérez Winter, C. (2013). Patrimonialización, turistificación y autenticidad en Exaltación de La Cruz (Argentina). Estudios y Perspectivas en Turismo, 22, 785-804. Recuperado a partir de http://www.estudiosenturismo.com.ar /PDF/V22/N04/v22n4a10.doc.pdf

Raffestin, C. (1986). Écogénèseterritoriale et territorialité. En F. Auriac, y R. Brunet (Dirs.). Espaces, jeux et enjeux (pp.175-185).París: Fayard.

Ricoeur, P. (2004). Finitud y culpabilidad. Madrid: Trotta.

Saarinen, J. (1998). The Social Construction of Tourist Destinations. The Process of Transformation of The Saariselka Tourism Region in Finnish Lapland. Destinations: Cultural landscapes of tourism, 1, 154-178. Recuperado a partir de https://www.taylorfrancis.com/books/e/9780203441381/chapters/10.4324/9780203441381-17

Santana Talavera, A. (2002). Mirar y leer. Autenticidad y patrimonio cultural para el consumo turístico. $6^{\circ}$ Encontro de Turismo con Base Local, Campo Grande, pp. 1-22.

Schenkel, E. (2012). Mito e Realidade: O Turismo Social como Primeira Política Peronista na Argentina. Revista Rosa dos Ventos, 4(4), 536-543.

Tarlow, P. y Mills, A. (1998). Turismo Extraterrestre y Hospitalidad: la cuarta dimensión. Estudios y Perspectivas en Turismo, 7, 258-269.

Wright, P. (2018). Periferias Sagradas en la Modernidad Argentina. Buenos Aires: Biblos.

\section{Notas}

1 En trabajos previos (Flores, 2011; Flores, 2016; Flores, 2018) hemos enfatizado la necesidad de romper con los esquemas dicotómicos que oponen al peregrino con el turista religioso (o cultural), e inclusive con los abordajes más gradualistas que proponen una transición entre aquellos visitantes que acuden por razones estrictamente religiosas, en un extremo, y otras exclusivamente culturales, en el otro.

2 El 9 de enero de 1986 apareció sobre el faldeo de la Sierra del Pajarillo, en la Quebrada de Luna, una superficie calcinada de forma oval de unos 100 metros de diámetro, que se denominó “Huella del Pajarillo". El hecho fue atribuido al descenso de un ovni y despertó el interés de turistas curiosos, de algunos pobladores locales y de investigadores del fenómeno ovni (Otamendi, 2018; Papalini, 2018).

3 En el universo cultural de la ovnilogía y sus seguidores, se denomina "contactismo" a la práctica a través de la cual determinadas personas pueden establecer diferentes formas de contacto (mental o física) con seres intra o extraterrestre o experimentar distintas manifestaciones de su presencia.

4 La experiencia se llevó a cabo en el mes de enero de 2020 en el Cerro Alfa de San Marcos Sierras.

5 En http://capilladelmontect.com.ar/ (última consulta enero de 2020). 
6 Este uso hegemónico del espacio no implica que no existan resistencias y contradicciones; por el contrario, se trata de un ámbito conflictivo donde se dan disputas por su definición, su uso y su control.

7 A pesar de ser esta narrativa "fundante", hay que rastrear algunos antecedentes unos años atrás, cuando a fines de los 70 comienzan a desarrollarse las primeras experiencias de contactismo, las ceremonias en Los Terrones llevadas a cabo por Ángel Acoglanis y las narrativas sobre la ciudad Erks, entre otras.

8 Se hace referencia al proceso mediante el cual un determinado sitio adquiere la particularidad de "lugar", esto es: un ámbito acotado con lógicas propias, cargado de sentidos e identificaciones, en relación y construcción con el afuera y atravesado por relaciones asimétricas de poder (Massey, 2004). En sintonía: "La práctica concreta da surgimiento auna mediación o estructura de sentimiento cultural (...) este sentido del lugarfortalece la definición socio-espacial del lugar y de lugarización (...); un lugar puede convertirse en un objeto deidentidad para un sujeto (o grupo), en su diferenciación con otros lugares" (Agnew, 1993, p. 13).

9 El cerro es propiedad de la familia Anchorena desde 1992 y está entregado en concesión para su explotación. Varias actividades se desarrollan en todo el complejo (incluido el ascenso) y recibe alrededor de 50 mil visitas al año. Cf. htt p://cerrouritorcoam.com.ar/.

10 Hacia el año 1999, la Municipalidad había instalado un cartel que señalaba la distancia del Uritorco hacia otros lugares, como Machu Picchu (Perú), Roswell (EEUU), Gizeh (Egipto), Stonehenge (Reino Unido), entre otros destinos considerados "esotéricos-energéticos" (Otamendi, 2006).

11 En https://www.eldiariodecarlospaz.com.ar/provincial/2020/2/6/capilla-del-monte-desalojara-losextraterrestres-deluritorco-81255.html?fbclid=IwAR1QYrJNUxw23tW4DKBC26qQVvgrEivjRZNnG7pRDVke5Ch2hJkqR3nJO4 (última consulta febrero de 2020).

12 En https://www.eldiariodecarlospaz.com.ar/provincial/2019/11/4/las-naves-otra-parte-peligra-el-festivalalienigena-d e-capilla-del-monte-75933.html (última consulta febrero de 2020).

13 En https://www.youtube.com/watch?v=-sT9ywvhaJg (última consulta febrero de 2020).

14 En https://www.facebook.com/fabriciodiazok (última consulta febrero de 2020).

15 Ángel Cristo Acoglanis decía ser griego,médico y que, mientras estabaen el Tíbet,había encontrado "la misión” y “el camino" en la espiritualidad. La realidad muestra que esta discursividad es parte del proceso de su invención como líder místico y constituye un componente central para entender sus discursos y sus prácticas. No había nacido en Grecia, sino en Rosario en 1924; no era médico (siempre trabajó en actividades informales) y nunca estuvo en el Tíbet. Para una biografía completa y muy bien documentada sobre Acoglanis, véase De Filippi (2018).

16 La estancia "La Aurora" (propiedad de la familia Tonna) es otro de los sitios ufológicos más destacados de América latina. Su sacralidad y su construcción como "lugar extraordinario" se funda sobre narrativas de contactos con seres extraterrestres y la ocurrencia de fenómenos paranormales desde los 70 .

17 Cf. http://losterrones.com.ar/ (última visita febrero de 2020).

18 "Un holograma espacial es un escenario situado en un lugar concreto y un tiempo igualmente demarcado, con lapeculiaridad de que en él están presentes otros lugares que actúan como constituyentes deese lugar” (Lindón, 2007, p. 42).

19 En http://www.hotelromacapilladelmonte.com/ (última consulta: febrero de 2020).

20 Entrevista a Osvaldo Allie, propietario del Hotel Roma y partícipe de las ceremonias (Capilla del Monte, enero de 2020). El propio Allie dice quepudoacompañarlo hasta allí en dos oportunidades, en las queAcoglanis le pidió que lo acompañase al interior de la ciudad de Erks. Dos veces ingresó Allie a este sitio etéreo

21. Por ejemplo, los Diarios de Erks que sistematizó Acoglanis, algunos audios de los mantras con su propia voz en Los terrones (https://www.youtube.com/watch?v=WLuIhxHV4zo) y los textos de Guillermo Terrera, José TrigueirinhoNetto y las fotografías de Roberto Villamil, entre otros.

22. Entrevista en la Agencia (enero de 2020). Todos los precios son para la temporada 2020.

23 Facebook Excursiones Angeluz (última consulta febrero de 2020).

24 Entrevista a Jonatan Mira (febrero de 2020).

25 Todas son expresiones vertidas en el trabajo de campo (enero de 2020).

26 Todas son expresiones provenientes del trabajo de campo (enero de 2020). 